\title{
CIE JOURNAL/REVUE ECI 34(1) \& 36(3) REFEREES/CRITIQUES
}

We would like to thank the many people to contribute their time and expertise to review articles for Volumes 34 to 36 of The Canadian and International Education Journal.

Nous voudrions remercier toutes les personnes qui ont contribué leur temps et leur expertise pour réviser les articles soumis à la Revue d'éducation canadienne et internationale, des volumes 34 au 36.

Anton Allahar
Jazira Asanova
Paul Barnard
K. P. Binda
Rethy Chhem
Nombuso Dlamini
Annabelle Dryden
Anthony Ezeife
Glen Eyford
Mokiko Fujikawa
Helen Harper
Yvonne Hébert
Daphne Heywood
France Jutras
Lucy Karanja
Rick Kitto
Larissa Klein

Melville Lall

Marianne Larsen

Stephane Levesque

Xuemei Li

Xiao Liang

Immaculate Namukasa

Jerry Paquette

Allan Pitman

Deo Poonwassie

David Radcliffe

Geri Salinitri

Kara Smith

Jeff Tennant

Teresa Van Deven

Aniko Varpalotai

Bing Wang

Sheri Zhang 